LPT Strasbourg 95-20

\title{
Local Fractional Supersymmetry for Alternative Statistics
}

\author{
N. Fleury* and M. Rausch de Traubenberg \\ Laboratoire de Physique Théorique, Université Louis Pasteur \\ 3-5 rue de l'université, 67084 Strasbourg Cedex, France \\ and \\ Centre de Recherches Nucléaires, Bât 40/II 67037 Strasbourg Cedex 2 \\ * IUFM d'Alsace- 200 route de Colmar, 67000 Strasbourg
}

\begin{abstract}
A group theory justification of one dimensional fractional supersymmetry is proposed using an analogue of a coset space, just like the one introduced in $1 D$ supersymmetry. This theory is then gauged to obtain a local fractional supersymmetry i.e. a fractional supergravity which is then quantized $\grave{a}$ la Dirac to obtain an equation of motion for a particle which is in a representation of the braid group and should describe alternative statistics. A formulation invariant under general reparametrization is given, by means of a curved fractional superline.
\end{abstract}


With string theory[1], a new approach came out in the description of space-time symmetry. Indeed, by studying the symmetries on the world sheet of the string one can get the space-time properties of the string states, i.e. the particles (representations of the gauge group are controlled by Kac-Moody algebra[2] and of the Poincaré one by (super)conformal invariance[3]). However, all those results were anticipated and an alternative formulation of relativistic wave equations [4, 5, 6] and quantum field theory can be obtained with the study of physics on the world line of the particle. Particles with spin $N / 2$ could be described by an $N$ - extented supersymmetry 6 on the world line, and gauge symmetries by the introduction of internal Grassmann variables [7]. All this was recently promoted into an alternative and efficient description of field theory using the world-line formalism [8], introducing $1 D$ Feyman rules and appropriate one dimensional Green functions $[9]$.

However, the spin statistics theorem and the Haag, Lopuszanski and Sohnius no-go theorem [10] tell us that supersymmetry is the more general non-trivial symmetry that one can consider; as soon as we are in a $D \leq 3$ dimensional space-time one can find statistics that are neither fermions nor bosons, but anyons 11] or particles which admit fractional statistics. Technically the former particles are in the representation of the permutation group and the latter of the braid group. In the meantime some extensions of $1 D$ supersymmetry have been considered, for instance parasupersymmetry [12, 13] or fractional supersymmetry 14, 15, 16, 17, 18]. It has been proved that $1 D$ parasupersymmetry of order $p$ could be equivalent to $p$-extended world-line supersymmetry and describes particles of spin $\frac{p}{2}[13]$.

Fractional supersymmetry has been recently the subject of intensive studies 14, 15, 16, 17, 18. Following the way which leads from $1 D$ supersymmetry to the Dirac equation, applied in the context of fractional supersymmetry, we get a new equation acting on states which are in the representation of the braid group. This equation can be seen as an extension of the Dirac equation in the sense that the $n$-th power of the field operator is equal to the Klein-Gordon one. In this paper we particularize the case $n=3$. In a first step we define, in analogy with the superspace, the fractional superspace as some kind of coset space reobtaining all what has been done in the framework of fractional susy. In a second step, we construct a local fractional supersymmetry i.e a fractional supergravity by using two one dimensional gauge fields: the einbein and a field which can be compared to the $1 D$ gravitino and that we call the fractional gravitino. A formulation, in a curved fractional superline, which is invariant under general coordinate transformations is then given. The second part is devoted to the quantization of the theory, taking under consideration the first and second class constraints 19. After having constructed the Fock space with the help of the $q$ - deformed oscillators [20] we obtain a new equation, that we call the fractional Dirac equation.

\section{I.Fractional Superspace and Fractional Supersymmetry}

Historically, 4D supersymmetry has been built explicitly, components by components (see for example [21]). However it was understood later that this symmetry is just a consequence of a symmetry in a so-called superspace which can be seen as the coset space of the Superpoincare group by the Lorentz group 21]. The superspace is just the 8 -fold space $\left(x^{\mu}, \theta\right)$, where $x^{\mu}$ is the space-time components and $\theta$ its spinor partner. Because we are studying physics on the world line we just particularize the $1 D$ case. Noting $H$ the generator of the time translation, and $Q$ the generator of the 
susy transformation, a point $(t, \theta)$ is parametrized by

$$
\exp (t H+\theta Q) \text {. }
$$

Using the susy algebra $[H, Q]=0,\{Q, Q\}=-2 H$ and the definition of a susy transformation with parameter $\epsilon$ we get the transformation law

$$
\begin{aligned}
\exp \left(t^{\prime} H+\theta^{\prime} Q\right) & =\exp (\epsilon Q) \exp (t H+\theta Q) \\
& =\exp ((t+i \epsilon \theta) H+(\theta+\epsilon) Q) .
\end{aligned}
$$

After having introduced the superfield $\Phi(t, \theta)=x(t)+i \theta \psi(t)$, it becomes easy to construct the susy transformation on the fields themselves and to build an invariant action.

Supersymmetry is the only nontrivial $Z_{2}$-extension of the Poincaré algebra 10] which is not in contradiction with the spin statistics theorem. However, in one dimension there is no obstruction to build other non trivial extensions. This is for instance parasupersymmetry 12, 13] or fractional supersymmetry 14, 15, 16, 17, 18]. The latter possesses a $Z_{n}$-structure, and through this article we will concentrate only on the $n=3$ case. Group theory justification of fractional supersymmetry (fsusy) has been undertaken in [17] but without the introduction of the analogue of the superspace, we call the fractional superspace (fsuperspace). A point in a fsuperspace is given by $(t, \theta)$, where $\theta$ is a real generalized Grassmann variable 22, 23, 24] of grade one submitted to the constraint $\theta^{3}=0$.

Let $Q$ be the generator of fsusy satisfying the condition

$$
Q^{3}=-H,
$$

and define a point in the fsuperspace by its parametrization

$$
\begin{aligned}
\exp _{g r}(t H+\theta Q) & =\exp _{g r}(t H) \exp _{g r}(\theta Q) \\
& =\exp (t H) \exp _{q}(\theta Q),
\end{aligned}
$$

where $e^{x p} p_{g r}$ is the graded exponential ( $t$ is of grade zero and $\theta$ of grade one), $q$ is a primitive cubic root of unity that we can take equal to $\exp (2 i \pi / 3)$ without losing generality and

$$
\exp _{q^{a}}(x)=\sum_{k=0}^{\infty} \frac{x^{n}}{\{n\}_{a} !},
$$

where $\{n\}_{a} !=\{n\}_{a}\{n-1\}_{a} \ldots\{1\}_{a},\{k\}_{a}=\frac{1-q^{a k}}{1-q^{a}}$.

This series exactly stops with its $(n-1)$-th power because $\theta^{n}=0$, in the general case. For $n=2$ we have only two terms and in this case the usual exponential coincides

* Some confusion exists between generalized Grassmann variables and Paragrassmann ones. Although, in the case of one variable those algebras coincide, they are different in general. The latter appears in the frame of parastastistics and is in some representation of the permutation group [25], whereas the former is just in a representation of the braid group 22, 23, 24 . 
exactly with the $\mathrm{q}(=-1)$-exponential and we recapture the definition (2). Going back to $n=3$ we get

$$
\exp _{q}(\theta Q)=1+\theta Q-q(\theta Q)^{2} .
$$

Now introduce $\epsilon$ the real parameter of the fsusy transformation $\left(\epsilon^{3}=0\right)$, and using the q-mutation relations (see the appendix for the justification of the q-mutators)

$$
\begin{aligned}
Q \theta & =q^{2} \theta Q \\
Q \epsilon & =q^{2} \epsilon Q \\
\theta \epsilon & =q \epsilon \theta,
\end{aligned}
$$

we get the fsusy transformation in the fsuperspace

$$
\begin{aligned}
\exp \left(t^{\prime} H\right) \exp _{q}\left(\theta^{\prime} Q\right) & =\exp _{q}(\epsilon Q) \exp (t H) \exp _{q}(\theta Q) \\
& =\exp \left(\left(t+q\left(\epsilon^{2} \theta+\epsilon \theta^{2}\right)\right) H\right) \exp _{q}((\theta+\epsilon) Q) .
\end{aligned}
$$

The transformations we have obtained coincide exactly with those of [17]. It has to be stressed again that $t^{\prime}=t+q\left(\epsilon^{2} \theta+\epsilon \theta^{2}\right)$ is real, as it should be. To obtain this equation we just developed explicitly eq.(8). The next step, to build an action, is to introduce a real fractional superfield $\Phi$ (fsuperfield) belonging to the fsuperspace. The Taylor expansion of $\Phi(t, \theta)$ gives

$$
\Phi(t, \theta)=x(t)+q^{2} \theta \psi_{2}(t)+q^{2} \theta^{2} \psi_{1}(t),
$$

where $x(t), \psi_{1}(t), \psi_{2}(t)$ are three real fields respectively of grade $0,1,2$ such that $\psi_{1}^{3}=$ $\psi_{2}^{3}=0$ and are submitted to the q-mutation relations (see the appendix)

$$
\begin{aligned}
\theta \psi_{1} & =q \psi_{1} \theta \\
\theta \psi_{2} & =q^{2} \psi_{2} \theta \\
\psi_{2} \psi_{1} & =q \psi_{1} \psi_{2} .
\end{aligned}
$$

It becomes now straightforward to obtain the transformations on the fsuperfield induced by fsusy transformations $\Phi(t, \theta) \longrightarrow \Phi\left(t^{\prime}, \theta^{\prime}\right)$. Inserting the values of $t^{\prime}, \theta^{\prime}$ obtained previously, we get the transformed fields :

$$
\begin{aligned}
\Phi\left(t^{\prime}, \theta^{\prime}\right) & =x\left(t^{\prime}\right)+q^{2} \theta^{\prime} \psi_{2}\left(t^{\prime}\right)+q^{2} \theta^{\prime 2} \psi_{1}\left(t^{\prime}\right) \\
& =x^{\prime}(t)+q^{2} \theta \psi_{2}^{\prime}(t)+q^{2} \theta^{2} \psi_{1}^{\prime}(t) \\
& =x(t)+q^{2} \epsilon \psi_{2}(t)+q^{2} \epsilon^{2} \psi_{1}(t) \\
& +q^{2} \theta\left(\psi_{2}(t)+\epsilon^{2} \dot{x}(t)-q \epsilon \psi_{1}(t)\right) \\
& +q^{2} \theta^{2}\left(\psi_{1}(t)+\epsilon \dot{x}(t)-q \epsilon^{2} \dot{\psi}_{2}(t)\right),
\end{aligned}
$$

implying the fsuperfield components transformations 14, 15, 16, 17, 18] 


$$
\begin{aligned}
\delta_{\epsilon} x & =q^{2} \epsilon \psi_{2} \\
\delta_{\epsilon} \psi_{2} & =-q \epsilon \psi_{1} \\
\delta_{\epsilon} \psi_{1} & =\epsilon \dot{x} .
\end{aligned}
$$

It has to be underlined that the transformed fields $x^{\prime}(t), \psi_{1}^{\prime}(t), \psi_{2}^{\prime}(t)$ do not satisfy the same q-mutation relations as the initial ones. To cure this problem in [16], a cocycle was introduced to correct the statistics. However, there is no need of such an object because the only fields that have to fulfill the same q-mutations as the initial ones are $x\left(t^{\prime}\right), \psi_{1}\left(t^{\prime}\right), \psi_{2}\left(t^{\prime}\right)$ and they $d o$. This is a quite general feature of quantum field theory. The reason why the new fields $x^{\prime}(t), \psi_{1}^{\prime}(t), \psi_{2}^{\prime}(t)$ do not actually fulfill the right q-mutation relations is that we have broken down explicitly, using Taylor expansion, the symmetry in the fsuperspace.

The next step is to construct a representation of the fsusy algebra acting on $\Phi$, as well as a covariant derivative to establish the action. We first need to recall some basic features of the derivation acting on generalized Grassmann variables. This structure, the q-deformed Heisenberg algebra, has been analyzed in[24] as well as its matrix representation [24, 26]. It admits in general $(n-1)$ derivatives, and we note $\partial_{\theta}$ and $\delta_{\theta}$ the two derivatives of the $n=3$ case which satisfy

$$
\begin{aligned}
\partial_{\theta} \theta-q \theta \partial_{\theta} & =1 \\
\delta_{\theta} \theta-q^{2} \theta \delta_{\theta} & =1 \\
\partial_{\theta}^{3}=0 \quad \delta_{\theta}^{3} & =0 \\
\partial_{\theta} \delta_{\theta}=q^{2} \delta_{\theta} \partial_{\theta} . &
\end{aligned}
$$

Then let us introduce the two basic objets of the fsusy $Q$ and $D$ the generator of fsusy and the covariant derivative respectively $14,15,16,17,18]$

$$
\begin{aligned}
Q & =\partial_{\theta}+q \theta^{2} \partial_{t} \\
D & =\delta_{\theta}+q^{2} \theta^{2} \partial_{t} .
\end{aligned}
$$

It can be checked explicitly that $D^{3}=Q^{3}=-\partial_{t}$ and $Q D=q^{2} D Q$. A direct calculation proves that

$$
\begin{aligned}
\Phi\left(t^{\prime}, \theta^{\prime}\right) & =\exp _{q^{2}}(\epsilon Q) \Phi(t, \theta) \\
\delta_{\epsilon} \Phi & =\epsilon Q \Phi(t, \theta) .
\end{aligned}
$$

Using the fact that $D$ q-mute with $Q$ we have $\delta_{\epsilon} D \Phi=D \delta_{\epsilon} \Phi$. Finally arguing that the $\theta^{2}$ component of $\Phi$ transforms like a total derivative we can take the opportunity to construct the action by taking the $\theta^{2}$ part of the action built in the fsuperspace. In other words, using the results on integration upon generalized Grassmann variables [27] $\int d \theta=\frac{d^{n}}{d \theta^{n}}$ we have

$$
\begin{aligned}
S & =-\frac{q^{2}}{2} \int d t d \theta \dot{\Phi} D \Phi \\
& =\int d t\left(\frac{x^{2}}{2}+\frac{q^{2}}{2} \dot{\psi}_{1} \psi_{2}-\frac{q}{2} \dot{\psi}_{2} \psi_{1}\right)
\end{aligned}
$$


So, from a pure group theoretical approach one gets the basic action usually used within the framework of fsusy 14, 15, 16, 17, 18. It can be pointed out that this action is real as it should be (for the q-mutators of the fields with the derivatives see the appendix).

To gauge these symmetries i.e to impose the invariance of the action under local diffeomorphism $t \longrightarrow t-f(t)$ and local fractional supersymmetry i.e fractional supergravity (fsugra) we need to introduce two real gauge fields $e$ the einbein and $\chi$ the fractional gravitino (fgravitino), that couple with their associated conserved charged $H=\frac{1}{2} \dot{x}^{2}$ for the diffeomorphism and $Q=\frac{q^{2}}{2}\left(\dot{x} \psi_{2}+\frac{1}{2} \psi_{1}^{2}\right)$ for the fsusy (S. Durand in 14]) respectively. Following the standard technics of gauge theory, noting $\pi=\dot{x}$, $\pi_{1}=\frac{q^{2}}{2} \psi_{2}$ and $\pi_{1}=\frac{-q}{2} \psi_{1}$ the conjugate momentum of $x, \psi_{1}, \psi_{2}$ and $H=\frac{1}{2} \pi^{2}$ the Hamiltonian, we have to replace $L=\pi \dot{x}+\dot{\psi}_{1} \pi_{1}+\dot{\psi}_{2} \pi_{2}-H$ by

$$
\begin{aligned}
L & =\pi \dot{x}-\frac{1}{2} e \pi^{2}+\dot{\psi}_{1} \pi_{1}+\dot{\psi}_{2} \pi_{2} \\
& +\frac{q^{2}}{4} \chi\left(\pi \psi_{2}+\frac{1}{2} \psi_{1}^{2}\right) \\
& +\frac{q}{4}\left(\psi_{2} \pi+\frac{1}{2} \psi_{1}^{2}\right) \chi .
\end{aligned}
$$

It is necessary to write, in the modified action, terms like $q^{2} \chi Q+q Q^{+} \chi$ to ensure the reallity of the new action. Taking the variation of the action with respect to $\pi$, we get

$$
\pi=\frac{\dot{x}}{e}+\frac{q^{2}}{2} \frac{\chi}{e} \psi_{2} .
$$

Inserting this value in the Lagrangian we finally obtain

$$
\begin{aligned}
L & =\frac{\dot{x}^{2}}{2 e}+\frac{q^{2}}{2} \dot{\psi}_{1} \psi_{2}-\frac{q}{2} \dot{\psi}_{2} \psi_{1} \\
& +\frac{q^{2}}{2} \chi\left(\frac{\dot{x}}{e} \psi_{2}+\frac{1}{2} \psi_{1}^{2}\right)-\frac{q^{2}}{4} \frac{\chi^{2}}{e} \psi_{2}^{2} .
\end{aligned}
$$

It is possible to rewrite this Lagrangian, introducing appropriate covariant derivatives, similarly to the spinning particles case 28.

$$
\begin{gathered}
D_{t} x=\dot{x}+\frac{1}{2} q^{2} \chi \psi_{2} \\
D_{t} \psi_{1}=\dot{\psi}_{1}+\frac{3}{2} \frac{1}{e} q \chi^{2} \psi_{2} \\
D_{t} \psi_{2}=\dot{\psi}_{2}-\frac{1}{2} q \chi \psi_{1} \\
L=\frac{1}{2 e} D_{t} x D_{t}^{+} x+\frac{1}{4}\left\{\left(q^{2} D_{t} \psi_{1} \psi_{2}+q \psi_{2} D_{t}^{+} \psi_{1}\right)\right. \\
-\frac{1}{4}\left(q D_{t} \psi_{2} \psi_{1}+q^{2} \psi_{1} D_{t}^{+} \psi_{2}\right) \\
=\int d \theta \frac{-q^{2}}{4}\left\{\left(\delta_{\theta}+q^{2} \theta^{2} \frac{1}{e} D_{t}\right) \Phi D_{t} \Phi+h . c .\right\}
\end{gathered}
$$


This action is a reminiscence of an action built in a curved fractional superspace in a way analogous to eq.(16). Like in the spinning particle case[何, we introduce the fractional einbein $E_{M}^{A}$, and its inverse $E_{A}^{M}$, where $M=\tau, \Theta, A=t, \theta$ are the curved/tangent indices of the fractional superline, which control the invariance by translation $\left(X^{M}=(\tau, \Theta) \longrightarrow X^{M}=X^{M}-\xi^{M}(X)\right)$. We restrict oursleves to affine transformations for $\Theta$, to ensure that it is invertible. Using the definition for integration on generalized grassmann variables we get $\int d \theta=\int d \theta^{\prime} J^{-2}$ with $J$ the Jacobian of the transformation. Then, following the way which leads to the superdeterminant [29] the transformations

$$
\tau^{\prime}=A \tau+B \Theta, \quad \Theta^{\prime}=C \tau+D \Theta
$$

give the following fractional superdeterminant $\operatorname{det}\left(A-B D^{-1} C\right) \operatorname{det}^{-2} D$ (for arbitrary $n$ we would have obtained $-n+1$ instead of -2$)$. With such a transformation we can build, using the fractional einbein, an invariant volume $S_{q} \operatorname{det}(E)=\left(E_{\tau}^{t}-\right.$ $\left.E_{\Theta}^{t} E_{\theta}^{\Theta} E_{\tau}^{\theta}\right)\left(E_{\theta}^{\Theta}\right)^{2}$. Doing so, we obtain the action

$$
S=-\frac{q^{2}}{4} \int d t d \theta S_{q} \operatorname{det}(E)\left(E_{t}^{M} \partial_{M} \Phi E_{\theta}^{N} \partial_{N} \Phi+h . c\right),
$$

with a huge invariance corresponding to the reparametrization of the fractional superline and possibly to transformations on the metric like in the spinning case[4]. The fsugra transformations, would correspond to a subset of these transformations with a special constrained choice of the parameters. Due to the Nother procedure the action (19) shall be invariant under this subset of transformations. Of course, one could whish to have an explicit formulation of the fsugra transformations. But this will be devoted to a future publication. To obtain the analogue of the Dirac equation, there is no need to know these transformation laws. What we need is just the local action (19). This action is invariant under the local diffeomorphism

$$
\begin{aligned}
\delta_{f} x & =f \dot{x} \\
\delta_{f} \psi_{1} & =f \dot{\psi}_{1} \\
\delta_{f} \psi_{2} & =f \dot{\psi}_{2} \\
\delta_{f} e & =f \dot{e}+\dot{f} e \\
\delta_{f} \chi & =f \dot{\chi}+\dot{f} \chi
\end{aligned}
$$

and under fsugra transformations.

\section{II.Dirac quantization}

Having obtained the full action which is invariant under fsupergravity transformation and one dimensional diffeomorphism, we are now in a position to quantize our

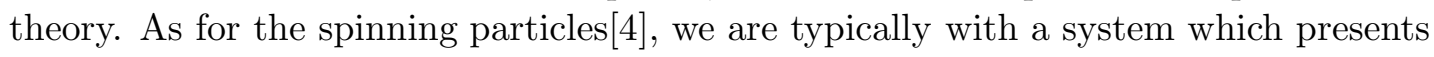
constraints. We have two second class constraints because the momenta of $\psi_{1}, \psi_{2}$ are not independent of the fields themselves 


$$
\begin{aligned}
& \Xi_{1}=\pi_{1}-\frac{q^{2}}{2} \psi_{2}=0 \\
& \Xi_{2}=\pi_{2}+\frac{q}{2} \psi_{1}=0
\end{aligned}
$$

and two first class constraints resulting of the gauge invariance of the $1 D$ fsugra and diffeomorphism

$$
\begin{aligned}
& \frac{\delta S}{\delta e}=H=\frac{1}{2} \pi^{2}=0 \\
& \frac{\delta S}{\delta \chi}=Q=\frac{q^{2}}{2}\left(\pi \psi_{2}+\frac{1}{2} \psi_{1}^{2}\right)=0
\end{aligned}
$$

so the einbein and the fgravitino just appear as Lagrange multiplier for the constraints. The quantization of a theory with constraints has been studied by Dirac 19], and a different treatment as to be implemented for first and second class constraints. For the first class ones we have to substitute the Poisson bracket to the Dirac one, and so the first step is to define an appropriate Poisson bracket for variables that q-mute. This can be done by using the q-symplectic metric 30 $\Omega$ or by noting that $\psi_{1}, \psi_{2}$ are in a representation of the quantum hyperplane 31 which admits a $R$-matrix convenient for such a construction ( see for example[32]). Recall that [30]

$$
\Omega=\left(\begin{array}{cccc}
0 & 1 & 0 & 0 \\
-q & 0 & 0 & 0 \\
0 & 0 & 0 & 1 \\
0 & 0 & -q^{2} & 0
\end{array}\right)
$$

where the indices $1,2,3,4$ are respectively for $\psi_{1}, \pi_{1}, \psi_{2}, \pi_{2}$ and that the $R$ - matrix stating $\psi_{2} \psi_{1}=q \psi_{1} \psi_{2}$ is 31

$$
R=\left(\begin{array}{cccc}
q^{2} & 0 & 0 & 0 \\
0 & q^{2}-q & 1 & 0 \\
0 & 1 & 0 & 1 \\
0 & 0 & 0 & q^{2}
\end{array}\right)
$$

With the use of $\Omega$ [30] or $R$ 32] we obtain

$$
\begin{aligned}
\{A, B\}_{P . B .} & =\frac{\partial A}{\partial x} \frac{\partial B}{\partial \pi}-\frac{\partial A}{\partial \pi} \frac{\partial B}{\partial x} \\
& +\frac{\partial A}{\partial \psi_{1}} \frac{\partial B}{\partial \pi_{1}}-q \frac{\partial A}{\partial \pi_{1}} \frac{\partial B}{\partial \psi_{1}} \\
& +\frac{\partial A}{\partial \psi_{2}} \frac{\partial B}{\partial \pi_{2}}-q^{-1} \frac{\partial A}{\partial \pi_{2}} \frac{\partial B}{\partial \psi_{2}}
\end{aligned}
$$

With this definition we can check explicitly that $\Xi_{1}, \Xi_{2}$ are second class constraints by calculating the algebra of the constraints

$$
\left\{\Xi_{i}, \Xi_{j}\right\}_{P . B .}=C_{i j}=\left(\begin{array}{cc}
0 & -q^{2} \\
q & 0
\end{array}\right)
$$


So following Dirac we define the Dirac bracket

$$
\{A, B\}_{D . B}=\{A, B\}_{P . B}-\left\{A, \Xi_{i}\right\}_{P . B} C_{i j}^{-1}\left\{\Xi_{j}, B\right\}_{P . B} .
$$

When we calculate the Dirac bracket of two $\psi$ 's and substitute the Dirac bracket by the q-mutator we obtain the quantized variables which satisfy

$$
\begin{aligned}
\psi_{1} \psi_{1}-\psi_{1} \psi_{1} & =0 \\
\psi_{2} \psi_{2}-\psi_{2} \psi_{2} & =0 \\
\psi_{2} \psi_{1}-q \psi_{1} \psi_{2} & =-q^{2} .
\end{aligned}
$$

This result is in exact accordance with the fact that the conjugate momentum of $\psi_{1}$ is $\frac{q^{2}}{2} \psi_{2}$ and it is wellknown that they have to belong to the q-deformed Heisenberg algebra 24] ( see for instance the q-mutator of $\theta, \partial_{\theta}$ ). With the quantized variables we directly check that the algebra (of the first class constraints) closes and we have $Q^{3}=H$ so the first class constraints are imposed upon the physical states

$$
H\left|\lambda_{\text {phys }}>=Q\right| \lambda_{\text {phys }}>=0 .
$$

To interpret these two equations we have to build the corresponding Fock space, but first we would like to have a formalism adapted to space-time i.e. when the variables carry space-time indices. So the space-time is just the target space in which the world-line is embedded. The full action (19), besides its $1 D$ dimensional invariance, is imposed to be $D$-dimensional Poincaré invariant. Noting $x^{\mu}, \psi_{1}^{\mu}, \psi_{2}^{\mu}$ the basic fields which are in the vectorial representation of the Poincaré group and $\eta_{\mu \nu}$ the Minkowski metric $\eta=\operatorname{diag}(1,-1, \cdots,-1)$ we have

$$
\begin{aligned}
L & =\frac{\dot{x}^{\mu} \dot{x}^{\nu}}{2 e} \eta_{\mu \nu}+\frac{q^{2}}{2} \dot{\psi}_{1}^{\mu} \psi_{2}^{\nu} \eta_{\mu \nu}-\frac{q}{2} \dot{\psi}_{2}^{\mu} \psi_{1}^{\nu} \eta_{\mu \nu} \\
& +\frac{q^{2}}{2} \chi\left(\frac{\dot{x}^{\mu}}{e} \psi_{2}^{\nu} \eta_{\mu \nu}+\frac{1}{2} \psi_{1}^{\mu} \psi_{1}^{\nu} \eta_{\mu \nu}\right)-\frac{q^{2}}{4} \frac{\chi^{2}}{e} \psi_{2}^{\mu} \psi_{2}^{\nu} \eta_{\mu \nu}
\end{aligned}
$$

Everything we have done up to now is suitable except that the product is replaced by a scalar product. The first question which arises concerns the q-mutation relations between the different components. Remember that we have various constraints

-(i) the fields have to be in a vectorial representation of the Poincaré group

-(ii) the fractional supercharge has to close the algebra:

$$
Q^{3}=\left(\pi^{\mu} \psi_{2}^{\nu} \eta_{\mu \nu}+\frac{1}{2} \psi_{1}^{\mu} \psi_{1}^{\nu} \eta_{\mu \nu}\right)^{3}=\frac{1}{2} \pi^{\mu} \pi^{\nu} \eta_{\mu \nu}
$$

with $\pi_{\mu}$ the conjugate momentum of $x^{\mu}$. This last relation is very strong and following the results of 23 on the linearization of polynomial( especially theorem 1.2 and its corollary and proposition $2.1,2.2$ ) the various components $\psi_{1}^{\mu}, \psi_{2}^{\nu}$ have to q-mute (see appendix for the q-mutations). However, those q-mutation relations are not stable under $S O(1, D-1)$ but only through the quantum group 31] $G L_{q}(D)$. So at a first glance it seems that (i) and (ii) are incompatible. However there is no need to impose the stability of the q-mutation relations under Lorentz transformations; so we just set the q-mutator 


$$
\psi_{a}^{\mu} \psi_{b}^{\nu}=q \psi_{b}^{\nu} \psi_{a}^{\mu}, \quad(a, \mu)<(b, \nu) \cdots a, b=1,2 ; \mu, \nu=0,1, \cdots D-1
$$

(the two possibilities of lexicographical order are defined in the appendix) in one special frame, called the q-frame or the q-gauge and by covariance if in this frame we have $Q^{3}=H$ it will be the case in any frame. It can be pointed out that a similar property appears in Yang-Mills theory, where the commutation relations of the components of the gauge field are not preserved under Lorentz transformations. Moreover, we see that the variables then obtained are not in a representation of the quantum hyperplane [31] because we have $\psi_{2}^{\mu} \psi_{1}^{\mu}-q \psi_{1}^{\mu} \psi_{2}^{\mu}=-q^{2}$.

With the $2 D$ variable $\psi_{a}^{\mu}$ we can, in this q-frame, define $D$ series of q-deformed oscillators [20] $a_{\mu}, a_{\mu}^{+}$

$$
\begin{aligned}
& a_{\mu}^{3}=a_{\mu}^{+3}=0 \\
& a_{\mu} a_{\mu}^{+}-q^{-1} a_{\mu}^{+} a_{\mu}=q^{N_{\mu}} \\
& a_{\mu} a_{\nu}=q a_{\nu} a_{\mu}, \quad \mu<\nu \\
& a_{\mu}^{+} a_{\nu}^{+}=q a_{\nu}^{+} a_{\mu}^{+}, \quad \mu<\nu \\
& a_{\mu}^{+} a_{\nu}=q^{+/-} a_{\nu} a_{\mu}^{+} .
\end{aligned}
$$

Where the $+/-$, in the last equation, corresponds to the two possible orderings (see appendix) and $N_{\mu}$ is the $\mu$-th number operator. With the $a_{\mu}^{+}$we can build a $3 D$ Fock space. If we note $\mid 0>$ the vacuum annihilated by all the $a$ 's we have

$$
\left|\lambda_{\text {phys }}>=\left(a_{0}^{+}\right)^{\alpha_{0}} \cdots\left(a_{D-1}^{+}\right)^{\alpha_{D-1}}\right| 0>, \quad \alpha_{D-1}, \cdots \alpha_{0}=0,1,2,
$$

and the relativistic wave $\left\langle x \mid \lambda_{\text {phys }}\right\rangle=\lambda(x)$ satisfies the wave equations

$$
\begin{aligned}
\partial_{\mu} \partial^{\mu} \lambda(x) & =0 \\
\left(i \psi_{2}^{\mu} \partial_{\mu}+\frac{1}{2} \psi_{1}^{\mu} \psi_{1 \mu}\right) \lambda(x) & =0 .
\end{aligned}
$$

Using a result of 23, that is, given a set of $k$ operators $A_{1}, \cdots A_{k}$ satisfying $A_{i} A_{j}=$ $q A_{j} A_{1}, i<j$ then $\left(\sum_{i=1}^{k} A_{i}\right)^{3}=\left(\sum_{i=1}^{k}\left(A_{i}\right)^{3}\right)$, we can easily prove that

$$
\left(i \psi_{2}^{\mu} \partial_{\mu}+\frac{1}{2} \psi_{1}^{\mu} \psi_{1 \mu}\right)^{3}=-\frac{1}{2} \partial_{\mu} \partial^{\mu} .
$$

This new operator can be seen as a cubic root of the d'Alembertian operator extending the Dirac equation to the fractional Dirac equation (fDirac), although the first equation tells us that we have a massless particle. Looking to equations (35), we just see that these new states are not in a representation of the permutation group but of the braid group. So we obtain states which constitute neither fermions or bosons nor parafermions or parabosons 25 but describe alternative statistics. Do we get anyons, but without Chern-Simon[11] terms, or fractional statistics? This is an open question. Of course such a representation is allowed for $D \leq 3$, to prove that $D$ is constrainted is still an open question. Some hint, to understand the meaning of (37), can be given. 
We can include, in our global Lagrangian, an additional term representing

- the interaction with an electromagnetic field. Note $g$ the coupling constant, $A_{\mu}(\Phi)$ the gauge field defined in the fsuperspace and $F_{\mu \nu}(x)$ the electromagnetic tensor

$$
\begin{aligned}
L_{e . m}= & -\frac{q^{2}}{2} \int d \theta\left(g A^{\mu}(\Phi) D \Phi_{\mu}+\text { h.c. }\right) \\
& =\quad g \dot{x}_{\mu} A^{\mu}+\frac{g}{2}\left(q \psi_{2}^{\mu} \psi_{1}^{\nu}+q^{2} \psi_{1}^{\mu} \psi_{2}^{\nu}\right) F_{\nu \mu} \\
& +\quad \frac{g}{4}\left(q^{2} \psi_{2}^{\alpha} \psi_{2}^{\beta} \psi_{2}^{\mu}+q \psi_{2}^{\mu} \psi_{2}^{\beta} \psi_{2}^{\alpha}\right) \partial_{\alpha} \partial_{\beta} A_{\mu} ;
\end{aligned}
$$

- as well as a coupling to the gravitational field. Note $g_{\mu \nu}(\Phi)=\eta_{\mu \nu}+h_{\mu \nu}(\Phi)$ the metric defined in the fractional superspace

$$
\begin{aligned}
L_{\text {grav. }} & =\frac{-q^{2}}{4} \int d \theta\left(\dot{\Phi}^{\mu} D \Phi^{\nu}+\text { h.c. }\right) h_{\mu \nu}(\Phi) \\
& =\frac{1}{2}\left(\dot{x}^{\mu} \dot{x}^{\nu}+q^{2} \dot{\psi}_{1}^{\mu} \psi_{2}^{\nu}-q \dot{\psi}_{2}^{\mu} \psi_{1}^{\nu}\right) h_{\mu \nu} \\
& +\frac{1}{4}\left(\left(-q^{2} \dot{x}^{\mu} \psi_{1}^{\nu} \psi_{2}^{\alpha}+q \dot{x}^{\mu} \psi_{2}^{\nu} \psi_{1}^{\alpha}+q \dot{\psi}_{2}^{\mu} \psi_{2}^{\nu} \psi_{2}^{\alpha}\right) \partial_{\alpha} h_{\mu \nu}+\text { h.c. }\right) \\
& \left.+\frac{1}{8}\left(q \dot{x}^{\mu} \psi_{2}^{\nu} \psi_{2}^{\alpha} \psi_{2}^{\beta}+\text { h.c. }\right) \partial_{\alpha} \partial_{\beta} h_{\mu \nu}+\text { h.c. }\right) .
\end{aligned}
$$

As a final remark we should notice that all this has nothing to do with the qdeformed spinning particles[30].

\section{Concluding remarks}

In a way similar to the one which has led to the description of the spinning particles, we have obtained, using local fsusy, a relativistic wave equation which describes states endowed with alternative statistics. The interpretation of what kind of particle we are describing is still an open question, just as the fact that the dimension $D$ cannot excess three. The case of the massive particles can be undertaken in a way similar to the one that has been introduced in the framework of $1 D$ susy [⿴囗口 , i.e. by the introduction of an auxiliary field. It should be interesting to extend all this formalism for any $n$. However, for that purpose we need a systematic way to obtain the local action, introducing a curved fractional superspace ( see eq. (22)) and paying attention to the symmetries of these action like for instance in the spinning particle case [4].

We have obtained these results using a one dimensional lagrangian formalism. However it should be possible to build similar actions for $D=2,3$. The case $D=2$ has been considered in an heterotic way [16], and in connection with string[33]. Those two actions should be analyzed within the framework of the fractional Virasoro 18 algebra and in connection with representation of the two dimensional Virasoro algebra.

As a final remark we just want to say that it is interesting to study all these kind of algebras in order to see how they can have connections with space-time properties. 


\section{Acknowledgement}

We would like to acknowledge J. -L. Jacquot and J. Polonyi for useful remarks and encouragments.

\section{Appendix}

In this appendix we just want to set up the q-mutation relations between the various fields and to see how they are arbitrary. We have two types of q-mutation relations, when the space-time indices are not involved and among the various components. First write the q-mutation without the space-time index.

$$
\begin{gathered}
\theta x=x \theta \\
\theta \psi_{1}=q \psi_{1} \theta \\
\theta \psi_{2}=q^{2} \psi_{2} \theta, \\
\partial_{\theta} x=x \partial_{\theta} \\
\partial_{\theta} \psi_{1}=q^{2} \psi_{1} \partial_{\theta} \\
\partial_{\theta} \psi_{2}=q \psi_{2} \partial_{\theta} .
\end{gathered}
$$

We have the same q-mutation with $\partial_{\theta}$ replaced by $\delta_{\theta}$.

$$
\begin{gathered}
\epsilon x=x \epsilon \\
\epsilon \psi_{1}=q \psi_{1} \epsilon \\
\epsilon \psi_{2}=q^{2} \psi_{2} \epsilon \\
\chi x=x \chi \\
\chi \psi_{1}=q \psi_{1} \chi \\
\chi \psi_{2}=q^{2} \psi_{2} \chi
\end{gathered}
$$

The relations (A.1-3) come from the definition of the grading, $\left(x, \psi_{1}, \psi_{2}\right)$ are of grading $0,1,2$ respectively. Of course the derivatives have the q-mutators with $q \longrightarrow q^{-1}$. We could have chosen equivalently the other cubic root $q^{2}$, which would have led to a substitution of $q \longrightarrow q^{-1}$ in all the q-mutation relations.

(A.7-9) are consequences of (A.1-3) due to the translation $\theta^{\prime}=\theta+\epsilon$ in the fsuperspace, they are also compatible with the fact that the variations on the fields under fsusy are real. (A.10-12) are consequences of the fsugra transformation ( from Nother theorem it is known that $\left.\delta_{\epsilon} \chi \sim \dot{\epsilon}\right)$.

$$
\begin{gathered}
\theta \epsilon=q \epsilon \theta \\
\theta \chi=q \chi \theta \\
\epsilon \chi=q \chi \epsilon
\end{gathered}
$$


(A.13) is imposed in order that the fsusy variations in the fsuperspace are real, (A.1415) results from (A.13).

$$
\begin{gathered}
\partial_{\theta} \theta-q \theta \partial_{\theta}=1 \\
\delta_{\theta} \theta-q^{2} \theta \delta_{\theta}=1 \\
\partial_{\theta} \delta_{\theta}=q^{2} \delta_{\theta} \partial_{\theta},
\end{gathered}
$$

by definition of the derivative in the q-deformed Heisenberg algebra.

$$
\begin{gathered}
\psi_{2} \psi_{1}=q \psi_{2} \psi_{1} \\
\dot{\psi}_{1} \psi_{1}=q^{a_{1}} \psi_{1} \dot{\psi}_{1} \\
\dot{\psi}_{2} \psi_{2}=q^{a_{2}} \psi_{2} \dot{\psi}_{2},
\end{gathered}
$$

(A.19) ensures the reality of the Lagrangian. A priori from $\psi_{i}^{3}=0$ we deduce that $\dot{\psi}_{i} \psi_{i}=q^{a_{i}} \psi_{i} \dot{\psi}_{i}$ but the choice of $a_{i}$ 's are arbitrary. Finally note that the derivative of the fields satisfy the same q-mutation relations as the field themselves.

After quantization (A.19) becomes

$$
\psi_{2} \psi_{1}-q \psi_{1} \psi_{2}=-q^{2} .
$$

To conclude we write the q-mutation relations when the space-time indices are involved

$$
\psi_{a}^{\mu} \psi_{b}^{\nu}=q \psi_{b}^{\nu} \psi_{a}^{\mu}, \quad(a, \mu)<(b, \nu),
$$

with $a, b=1,2$ and $\mu, \nu=0,1,2$. Two possible orderings are allowed

(1) $(a, \mu)<(b, \nu)$ if $\mu<\nu$ else $\mu=\nu$ and $a<b$;

(2) $(a, \mu)<(b, \nu)$ if $a<b$ else $a=b$ and $\mu<\nu$.

And only (2) allows a matrix representation. With the notations of 26] we have $(D=3$ and for an Euclidian space, or after a Wick rotation)

$$
\begin{gathered}
\psi_{1}^{0}=\theta \otimes \sigma_{3} \otimes \sigma_{3} \\
\psi_{1}^{1}=I_{3} \otimes \theta \otimes \sigma_{3} \\
\psi_{1}^{2}=I_{3} \otimes I_{3} \otimes \theta, \\
\psi_{2}^{0}=-q^{2} \partial_{\theta} \otimes \sigma_{3}^{2} \otimes \sigma_{3}^{2} \\
\psi_{2}^{1}=-q^{2} \cdot I_{3} \otimes \partial_{\theta} \otimes \sigma_{3}^{2} \\
\psi_{2}^{2}=-q^{2} \cdot I_{3} \otimes I_{3} \otimes \partial_{\theta},
\end{gathered}
$$

with

$$
\begin{gathered}
\theta=\left(\begin{array}{lll}
0 & 0 & 0 \\
1 & 0 & 0 \\
0 & 1 & 0
\end{array}\right) \\
\partial_{\theta}=\left(\begin{array}{ccc}
0 & 1 & 0 \\
0 & 0 & 1+q \\
0 & 0 & 0
\end{array}\right)
\end{gathered}
$$




$$
\sigma_{3}=\left(\begin{array}{ccc}
1 & 0 & 0 \\
0 & q & 0 \\
0 & 0 & q^{2}
\end{array}\right) .
$$

$\theta, \partial_{\theta}$ are the matrices which appear in the literature of the $q$-deformed Heisenberg algebra [24, 26], $\sigma_{3}$ is one of the basic matrix appearing within the framework of generalized Clifford algebra( see[23] and references therein) and $I_{3}$ is the $3 \times 3$ indentity matrix.

\section{References}

[1] M. B. Green, J. H. Schwarz and E. Witten, Superstring theory, Cambridge Univ. Press, 1987.

[2] P. Goddard and D. Olive, Int. J. Mod. Phys. A1 (1986) 303.

[3] A. A. Belavin, A. M. Polyakov and A. B. Zamolodchikov, Nucl. Phys. B241 (1984) 333.

[4] L. Brink, S. Deser, B. Zumino, P. Di Vecchia and P. Howe, Phys. Lett. 64B (1976) 435; L. Brink, P. Di Vecchia and P. Howe, Nucl. Phys. B118 (1977) 76.

[5] R. Casalbuoni, Phys. Lett. 62B (1976) 49; R. Casalbuoni, Nuovo Cimento 33A (1976) 389; F. A. Brezin and M. S. Marinov, Ann. Phys. 104 (1977) 336; M. Henneaux and C. Teitelboim, Ann. Phys. 143 (1982) 127; V. Y. Fainberg and A. V. Marshakov, Nucl. Phys. B306 (1988) 659.

[6] P. Howe, S. Penati, M. Pernici and P. Townsend Phys. Lett B215 (1988) 555.

[7] A. P. Balachandran, P. Salomonson, B.-S. Skagerstam and J.-O. Winnberg, Phys. Rev. D15 (1977) 2308; A. Barducci, R. Casalbuoni and L. Lusanna Nucl. Phys. B124 (1977) 93.

[8] A. M. Polyakov, Gauge Fields and Strings, Harwood, 1987; Z. Bern and D. A. Kosower, Nucl. Phys. B379 (1992) 451; M. J. Strassler, Nucl. Phys. B385 (1992) 145.

[9] M.G. Schmidt and C. Schubert Phys.Lett. B331 (1994) 69; Multiloop calculations in QED by Superparticle Path Integrals Talk given at High Energy Physics: QCD Workshop 94, Montpellier, France, 7-13 Jul 1994, hep-ph/9408394.

[10] R. Haag, J. T. Lopuszanski and M. F. Sohnius Nucl. Phys. B88 (1975) 257.

[11] A. Lerda Quantum Mechanics of Particles with Fractional Statistics,1992, Springer, Berlin.

[12] V. A. Rubakov and V. P. Spiridonov Mod. Phys. Lett. A3 (1988) 1332; J. Beckers and N. Debergh Mod. Phys. Lett. A4 (1989) 1209; S. Durand, R. Floreanini, M. Mayrand and L. Vinet Phys. Lett. B233 (1989) 158; A. Khare J. Phys. A25(1992) L1749; N. Fleury, M. Rausch de Traubenberg and R. M. Yamaleev Int. J.Mod. Phys. A10 (1995) 1269.

[13] G. P. Korchemsky Int. J.Mod. Phys. A7 (1992) 3493.

[14] C. Ahn and D. Bernard Nucl. Phys. B346 (1990) 409; S. Durand Phys. Lett B312 ( 1993) 115; S. Durand Mod. Phys. Lett A8 ( 1993) 2323; N. Debergh J. Phys. A26 (1993) 7219. 
[15] J. L. Matheus-Valle and Marcos A. R. Monteiro Mod. Phys. Lett. A7 (1992) 3023; L. P. Collatto and J. L. Matheus-Valle On q-deformed classical mechanical models ICTP- Trieste, preprint april 1995.

[16] J. L. Matheus-Valle and Marcos A. R. Monteiro Phys. Lett. B300 (1993) 66.

[17] J. A. de Azcárraga and A. J. Macfarlane Group Theoretical Foundations of Fractional Supersymmetry, preprint FTUV/95-23 and IFIC/95-23, hep-th/9505177.

[18] S. Durand Mod. Phys. Lett A7 ( 1992) 2905.

[19] P. A. M. Dirac Lecture on Quantum Mechanics, Belfer Graduate School of Science, Yeshiva University, New-York 1964.

[20] A. J. Macfarlane J. Phys. A22 (1989) 4581; L. C. Biedenharn J. Phys. A22 (1989) L873.

[21] See e.g. J. Wess and J. Bagger Supersymmetry and Supergravity, Princeton Series in Physics,Princeton Univ. Press 1983, Princeton, New-York.

[22] A. K. Kwasniewski J. Math. Phys. 26 (1985) 2234.

[23] N. Fleury and M. Rausch de Traubenberg J. Math Phys (1992) 33; Adv. Appl. Clif. Alg. 4 (1994) 123.

[24] A. T. Filipov, A. P. Isaev and R. D. Kurdikov Mod. Phys. Lett. A7 (1992) 2129.

[25] H. S. Green Phys. Rev 90 (1952) 270; Y. Ohnuki and S. Kamefuchi Quantum Field Theory and Parastistics, Springer-Verlag, Berlin, Heidelberg, New-York (1982).

[26] M. Rausch de Traubenberg Adv. Appl. Clif. Alg. 4 (1994) 131.

[27] N. Fleury and M. Rausch de Traubenberg Beyond Spinors, in Leite Lopes Festschrift p. 79, World Scientific 1988, Singapore; L. Beaulieu and E. G. Floratos Phys. Lett. B258 (1991) 171.

[28] J. W. van Holten $D=1$ Supergravity and Spinning Particles, preprint NIKHEF/95055 hep-th/9510021.

[29] F. A. Berezin Introduction to Superanalysis, edited by A. A. Kirillov, D. Reidel Pub. Company,1987, Dordecht.

[30] R. P. Malik Phys. Lett. B316 (1993) 257.

[31] J. Wess and B. Zumino Nucl. Phys. B (proc. Suppl.) 18B (1990) 302.

[32] See e.g J. Fuchs Affine Lie algebras, Quantum Groups: an introduction, with applications in Conformal Field Theory Cambridge Univ. Press (Cambridge monographs on mathematical physics), 1992.

[33] T. Nakanishi Mod. Phys. Lett A3 (1988) 1507; Progr. Theor. Phys. 82 (1989) 207. 